\title{
A FPN Parameter Optimization Method Based on CDEDPSO
}

\author{
Qiumin $\operatorname{Fan}^{1, a}$, Bing $X u^{2, b}$ \\ ${ }^{1}$ Shanghai Institute of Technology(Haiquan Road N.100,Fengxian District,Shanghai), China \\ ${ }^{2}$ Shanghai Institute of Technology(Haiquan Road N.100,Fengxian District, Shanghai), China \\ a1759554064@qq.com, bubing@sit.edu.cn
}

\begin{abstract}
Keywords:optimization algorithm; FPN; PSO
Abstract. For the uncertain change characteristics of transition matrix parameters in complex FPN system,this paper presents a parameters optimization algorithm based on CDEDPSO.This article focuses on the weight, threshold and certainty of FPN optimized model and these parameters are analyzed, improved and simulated simultaneously. This method implements dynamic programming and self-learning of uncertain characterization parameters in FPN.Finally, in this paper, the method is applied to a FPN model and the feasibility and effectiveness of this method has been verified.
\end{abstract}

\section{Introduction}

The transition matrix in FPN model is a collection of trigger condition parameters and the biggest drawback is the poor self-learning ability for the uncertain parameters. The changes feature of uncertain parameters are reflected in these areas:the determination of weight, threshold, certainty and other parameters. Therefore, Consider how to deal with and improve uncertain parameters of FPN to optimize FPN uncertain knowledge representation model,enhance the dynamic programming and self-learning ability of FPN and improve the efficiency and accuracy of FPN model has became an important research topic ${ }^{[1]}$.

In reference[1], it uses GPSO algorithm to optimize uncertain parameters and to some extent, it has improved the solution.Based on expert system,reference[2] has established FPN uncertain knowledge representation model and enhanced the practicability of the algorithm.However, in terms of accuracy of FPN, these methods are lacking.According to the reference[1,2], this article has further improved the performance of optimization algorithm.It makes FPN dynamic programming and self-learning ability improved and enhances the efficiency and accuracy of FPN.In addition, it provides a new solution for the changes of uncertain parameters in FPN.

\section{FPN Knowledge Representation Rules}

\section{Optimization Problem}

The matrix of FPN reasoning model is the form of a ten-tuple ${ }^{[3]}$ :

$$
F P N=\{P, T, D, I, O, M, T h, W, f, \beta\}
$$

In the formula (1), $P=\left\{p_{1}, p_{2}, \ldots, p_{n}\right\}$ represents place matrix; $T=\left\{t_{1}, t_{2}, \ldots, t_{m}\right\}$ represents transition matrix, and transition matrix is composed by a number of knowledge representation model; $D=\left\{d_{1}, d_{2}, \ldots, d_{n}\right\}$ represents a finite set of propositions; I represents the input matrix;O represents the output matrix; $\mathrm{M}: \mathrm{P} \rightarrow[0,1]$, it is an identity function, assign an identification $M\left(p_{i}\right)$ to place $p_{i} \in \mathrm{P}$, represents the true value of the place corresponding fuzzy proposition; Th: $\mathrm{T} \rightarrow[0,1]$,define a threshold value $T h(t)=\lambda$ for transition $(\mathrm{t} \in \mathrm{T}) ; W=\left\{\omega_{1}, \omega_{2}, \ldots, \omega_{r}\right\}$ represents the weight vector of knowledge rules, $0 \leq \omega_{j} \leq 1$ and $\sum_{j=1}^{r} \omega_{j}=1$; f: $\mathrm{T} \rightarrow[0,1]$, represents the certainty of rules that transition given, $f(t)=\mu ; \beta: \mathrm{P} \rightarrow \mathrm{D}$, it used to correspond transition node and 
propositions.

FPN knowledge representation rules are generally divided into two categories ${ }^{[4]}$ :

Type 1: if $d_{1}$ and $d_{2}$ and...... and $d_{n}$ then $d\left(\mu, \lambda, \omega_{1}, \omega_{2}, \ldots \omega_{r}\right)$;

Type 2: if $d_{1}$ or $d_{2}$ or..... or $d_{n}$ then $\mathrm{d}(\mu, \lambda)$;

Among them: $d_{i}(i=1,2, \ldots, n)$ is premise proposition, $d$ is the result of proposition, $\mu$ is the certainty of the rules, $\lambda$ is the application threshold of rules, $\omega_{j}(j=1,2, \ldots, r)$ is weight.

Transition $T=\left\{t_{1}, t_{2}, \ldots, t_{m}\right\}$ is a set of transition of reasoning model.Suppose transition $\mathrm{T}$ has $\mathrm{n}$ input places, then in the reasoning process, the algorithm of new identity value generated by transition $\mathrm{t}$ is as follows:

When the condition is satisfied $\forall p_{j} \in I(t)$,

$$
z(t)=\left\{\begin{array}{cl}
f(t) \bullet \sum_{j=1}^{n}\left[M\left(p_{j}\right) \bullet \omega_{j}\right], & \sum_{j=1}^{n}\left[M\left(p_{j}\right) \bullet \omega_{j}\right] \geq T h(t) \\
0, & \sum_{j=1}^{n}\left[M\left(p_{j}\right) \bullet \omega_{j}\right]<T h(t)
\end{array}\right.
$$

In formula 2, the identity value is not easy to learn from uncertain parameters because the calculation and maximum operation of identity values are not continuous. Therefore, we need to calculate the value of a continuous function simulation mark.In reference, discontinuous sign function can be replaced by continuous S-function ${ }^{[5]}$.The expression of S-type functions are as follows:

$$
y(x)=1 /\left(1+e^{-h(X-k)}\right)= \begin{cases}1, & X \geq k \\ 0, & X<k\end{cases}
$$

Among them, $\mathrm{h} \rightarrow \infty$ is a constant.Using S-type function analog identification value calculated as follows:

Suppose $X=\sum_{j=1}^{n} M\left(p_{j}\right) \bullet \omega_{j}, k=T h(t)$, the identity value that transition $\mathrm{t}$ stimulate generated is as follows:

$$
z(t)=f(t) \bullet X /\left(1+e^{-h(X-T h(t))}\right)=\left\{\begin{array}{cc}
f(t) \bullet X, & X \geq T h(t) \\
0, & X<T h(t)
\end{array}\right.
$$

In formula $4, z(t) \in[0,1]$.

Definition 1 Uncertain parameters of FPN means:certainty $f(t)=\mu$, the weight of knowledge representation rules $\omega_{\mathrm{j}}(\mathrm{t})$, transition trigger threshold $\mathrm{TH}(\mathrm{t})=\lambda$. Transition uncertainty means: since the identity value of transition affected by uncertain parameters, transition trigger conditions have random uncertainty.

So how to optimize uncertain parameters of reasoning model transition matrix to increase the dynamically adapt and self-learning ability of FPN model for the change of uncertainty parameters and to improve the accuracy of FPN is an urgent problem.

\section{Optimization function of the model parameters}

Reasoning based on FPN is beginning with the initial identification,all transitions meet the conditions in order to stimulate parallel and produce new identity to the output place.Then, the place token will be transferred to the target until the end of the run. Termination place identification value is the final result of reasoning.

For the place $\mathrm{P}$ that have $\mathrm{m}$ input transition,after all transitions are excited at the same time, the identity value $M(p)$ of the place is the maximum identity value among these transitions, and that is: 
$M(p)=\max \left\{z\left(t_{1}\right), z\left(t_{2}\right), \ldots, z\left(t_{m}\right)\right\}$

Among them, $M(p) \in[0,1], z(t)$ is the identity value of t.Similarly, the actual identity value of the place can be expressed as:

$$
M(p)=\max \left\{z\left(t_{k+1}\right), z\left(t_{k}\right)\right\} \cong z\left(t_{k+1}\right) /\left(1+e^{-h\left(z\left(t_{k+1}\right)-z\left(t_{k}\right)\right)}\right)+z\left(t_{k}\right) /\left(1+e^{-h\left(z\left(t_{k}\right)-z\left(t_{k+1}\right)\right)}\right)
$$

Error cost function can be characterized the random uncertainty of FPN transition matrix parameters $^{[6]}$. So it can take advantage of the error cost function value to reflect the strength of uncertainty parameters. The error cost function is as follows:

$E=\frac{1}{2} \sum_{i=1}^{r} \sum_{j=1}^{b}\left(M_{i}\left(p_{j}\right)-M_{i}^{\prime}\left(p_{j}\right)\right)^{2}$

In formula $7, \mathrm{~b}$ is the number of the termination place, $\mathrm{r}$ is the number of samples, and $b, r \in \mathrm{N}$, $M_{i}\left(p_{j}\right), M_{i}^{\prime}\left(p_{j}\right)$ represent actual identity values and expected identity values of the $\mathrm{i}$-th sample of termination place $p_{j}$. The expected identity values will follow principles: if the rule is expected to be occurred,then $M_{i}^{\prime}\left(p_{j}\right)=1$, or $M_{i}^{\prime}\left(p_{j}\right)=0$.The value of $\mathrm{E}$ is proportional to the strength of the model uncertainty.

Definition 2 If there is transition $t_{1}$,place $p \in I\left(t_{1}\right)$, but there is not transition $t_{2}$,place $p \in O\left(t_{2}\right)$, then call place $\mathrm{p}$ initial place; If there is transition $t_{1}$, place $p \in O\left(t_{1}\right)$, but there is not transition $t_{2}$,place $p \in I\left(t_{2}\right)$, then call place $\mathrm{p}$ termination place.

Therefore, the purpose of optimization is to make the deviation of uncertain parameters tend to the smallest.Therefore, the optimization objective function in this article is as follows:

$$
F(t)=\min (E)=\min \left(\frac{1}{2} \sum_{i=1}^{r} \sum_{j=1}^{b}\left(M_{i}\left(p_{j}\right)-M_{i}^{\prime}\left(p_{j}\right)\right)^{2}\right)
$$

\section{Optimization Algorithm of Error Cost Function}

The traditional evolutionary algorithm are BP and PSO algorithm,but BP algorithm has complex iterated function and a large error ${ }^{[7]}$.PSO algorithm need not to take derivative of error cost function, shorten the solution time and avoid the error generated by derivation. But the standard PSO algorithm prone to 'premature' phenomenon. So it is necessary to improve the standard PSO to improve the accuracy and efficiency of the algorithm.

\section{DPSO algorithm principle}

PSO is an algorithm to seek the optimal solution both based on groups and individual collaboration.In the PSO algorithm, each particle represents a possible solution. The optimal position each particle have experienced is called pbest ( current best position). The best position the entire group had experienced is called gbest (global best position).During the flight,particle determine the next state of motion based on their experience and the best experience of companion.During the optimization process of particle, the trajectory of $\mathrm{N}$-th particle is as follows:

$$
\begin{aligned}
& v_{i(t+1)}=\omega v_{i}(t)+r_{1} c_{1}\left(\text { pbest }-x_{i}(t)\right)+r_{2} c_{2}\left(\text { gbest }-x_{i}(t)\right) \\
& x_{i}(t+1)=x_{i}(t)+v_{i}(t+1)
\end{aligned}
$$

In formula $9, r_{1}$ and $r_{2}$ are random numbers between 0 and $1 ; c_{1}$ and $c_{2}$ are acceleration constants; $\omega$ is inertia weight; When $\omega$ is large, the algorithm has strong global search capability. When $\omega$ was smaller,the algorithm tends to local search.

The traditional PSO algorithm converges faster, but the particles are likely to occur "premature" phenomenon prematurely and it will affect the optimization performance of algorithm significantly.In references, in order to improve the premature convergence of algorithm, it uses 
random perturbation strategy for particle swarm.And the method avoid particles premature effectively.However, the method used a single uniform mutation mode during the mutation operation and it weakened the ability of particles to escape.It leads to less change in particle diversity and global optimization ability is not strong.For the shortcomings above,this paper presents a follow mutation particle perturbations changing inertia weight PSO (DPSO) algorithm. The algorithm introduces a mutation of particles and it will associate particle population consistent trends with particle mutation. The basic idea is:in the optimization process, once the particles are consistent, the particle will be disturbed and the particles will mutate in order to maintain the diversity of particles within the search space.It avoids the particles fall into local optimization and eliminates 'premature' of algorithm as soon as possible.Meanwhile, according to the degree of variation of the particle, inertia weight to be adjusted accordingly. So that the improved algorithm is more stable in the global search capability ${ }^{[8]}$.

1)Particles tend consistency determination method

This article determine whether particles converge according to the similarity of particle dispersion position. The specific method is to determine whether particles convergence based on the average variance of particle dispersion $x_{i}$. The formula is expressed as follows:

$d=\left(\frac{1}{k} \sum_{i=1}^{k}\left(x_{i}-\overline{x_{i}}\right)^{2}\right)^{1 / 2}, i=1,2, \ldots, k$

In formula $11, \mathrm{k}$ is the number of particles; $\mathrm{d}$ represents the average variance of particle dispersion. When $\mathrm{d}$ is less than a certain threshold, indicating the particles converge.

2)Particle mutation approach

When the particles converge and occur 'premature' phenomenon, the algorithm is very likely to fall into local optimal state.In this regard, we introduce mutation thought.That is applying perturbation to the current state of the particle within the search range.Its perturbation operating can be expressed as:

$x_{\text {inew }}=x_{\text {iold }}+\delta$

In formula 12, $\delta$ represents a random disturbance; $x_{\text {iold }}$ represents the state of the particle before the mutation; $x_{\text {inew }}$ represents the state of the particle after the mutation.

3)Inertia weight change approach

This article will use the method that follow mutation particle perturbation degree to adjust the value of inertia weight.It makes the algorithm more stable in the global search capability and accelerates the convergence rate of single iteration optimization algorithm. The formula is as follows:

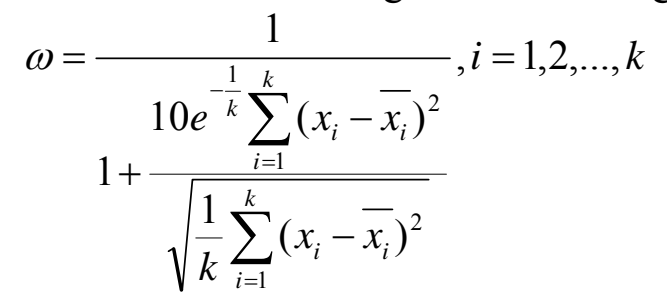

As can be seen from the above formula,the value of $\omega$ changes between 0 and 1 . When the particles converges, $\omega$ decreases.In this case, it has particles mutation to increase the diversity of particles. While adjusting the value of $\omega$ to make it operate following the mutation particle in order to the global optimization state once again.

\section{DE algorithm principle}

DPSO algorithm has a fast convergence and robustness and it is able to handle the particle precocious problem as soon as possible.But its global search performance needs to be improved.Therefore, this paper introduces DE algorithm based on this algorithm.DE algorithm can improve the diversity of the population further and it has a strong global search capability ${ }^{[9]}$. 
DE algorithm implements individual variability through differential mutation strategy.DE algorithms include mutation, crossover and selection operations.

1)Mutation

This paper uses DE/current-to-best/bin differential strategy ${ }^{[10]}$ :

$V_{i}(t+1)=X_{i}(t)+\lambda \bullet\left(X_{\text {best }}(t)-X_{i}(t)\right)+F \bullet\left(X_{r 1}(t)-X_{r 2}(t)\right)$

Among them, $r_{1}, r_{2}, r_{3} \in\{1,2, \ldots, n\}$ and $i \neq r 1 \neq r 2, \lambda, \mathrm{F}$ is scaling factor; $X_{\text {best }}(t)$ is the best individual in the population of generation $\mathrm{t} ; X_{i}(t)$ is the $\mathrm{i}$-th individual of population in generation t.The advantage of this differential strategy is that it can use $\left(X_{\text {best }}(t)-X_{i}(t)\right)$ to improve the convergence rate and also can use $\left(X_{r 1}(t)-X_{r 2}(t)\right)$ to increase the diversity of population.

2)Crossover

In cross-operation, new population $y_{i}(t)=\left[y_{i 1}(t), y_{i 2}(t), \ldots, y_{i 3}(t)\right]$ co-produced by the random vector $v_{i}=\left[v_{i 1}, v_{i 2}, \ldots v_{i D}\right]$ and target vector $x_{i}=\left[x_{i 1}, x_{i 2}, \ldots, x_{i D}\right]$.

$y_{i j}(t)= \begin{cases}v_{i j} & (\operatorname{randb}(j)<=C R) \operatorname{or}(j=\operatorname{randr}(i)) \\ x_{i j} & (\operatorname{randb}(j)>C R) \operatorname{and}(j \neq \operatorname{randr}(i))\end{cases}$

In formula $15: j \in[1, D] \operatorname{rand}(j) \in[0,1]$ is the $j$-th value of the same random number generator. $C R \in[0,1]$ is mutation probability. $\operatorname{randr}(i) \in[1,2, \ldots, D]$ is random selection index and it guarantees that $v_{i}(t)$ provides at least one parameter to $x_{i}(t)$.

3)Selection

In selecting operation, the paper uses greedy strategy. That means only when the offspring is superior to the parent, the offspring will be retained,otherwise be eliminated.

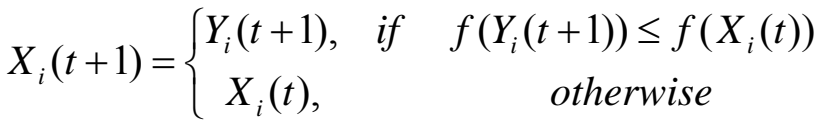

Hybrid optimization algorithm

In order to enhance the performance of the algorithm to a greater extent, this paper takes particle premature judgment mechanism for judging the degree of aggregation of the particles based on DPSO.The aim is to determine whether the DE algorithm needs to reduce the degree of aggregation of the particles and increase the diversity of the population. The calculation method of premature judgment mechanism is as follows:

Suppose the number of particles in particle swarm is $\mathrm{N}, f_{i}$ is fitness of the $\mathrm{i}$-th particle, $f_{\text {avg }}$ is the current average fitness of particle swarm and the fitness variance of particle swarm can be defined as $\sigma^{2}:$

$\sigma^{2}=\frac{1}{N} \sum_{i=1}^{N}\left(\frac{f_{i}-f_{\text {avg }}}{f}\right)^{2}$

In formula $17, \mathrm{f}$ is the normalized scaling factor and the role is to limit the size of $\sigma^{2}$.Values of $\mathrm{f}$ need to meet the following conditions:after normalization, the maximum value of $\frac{\left|f_{i}-f_{\text {avg }}\right|}{f}$ is not more than 1 ; The value of $\mathrm{f}$ changes with evolutionary algorithm. $\sigma^{2}$ reflects the degree of aggregation of the particles and the larger the value, the smaller the degree of aggregation and the stronger global search ability;Conversely, the weaker.Generally,compare $\sigma^{2}$ and $\sigma_{T}^{2}$ (population fitness variance threshold).If $\sigma^{2}<\sigma_{T}^{2}$, it indicates the degree of aggregation of particle group is too high and then we need to take measures to change the degree of aggregation of particle group.The aim is to improve the global search ability of groups. Since the initial population in PSO algorithm is 
randomly generated and it is not representative,so this article dose a chaos initialization for initial population to ensure the diversity of the initial population.Chaos algorithm has some characteristics like the randomness,ergodicity and regularity and so on.This method does not change the randomness of initial population, and improves the diversity of the population using the ergodicity of chaotic algorithm. This helps to find the optimal solution ${ }^{[11]}$.

CDEDPSO algorithm flow chart shown in Figure 1:

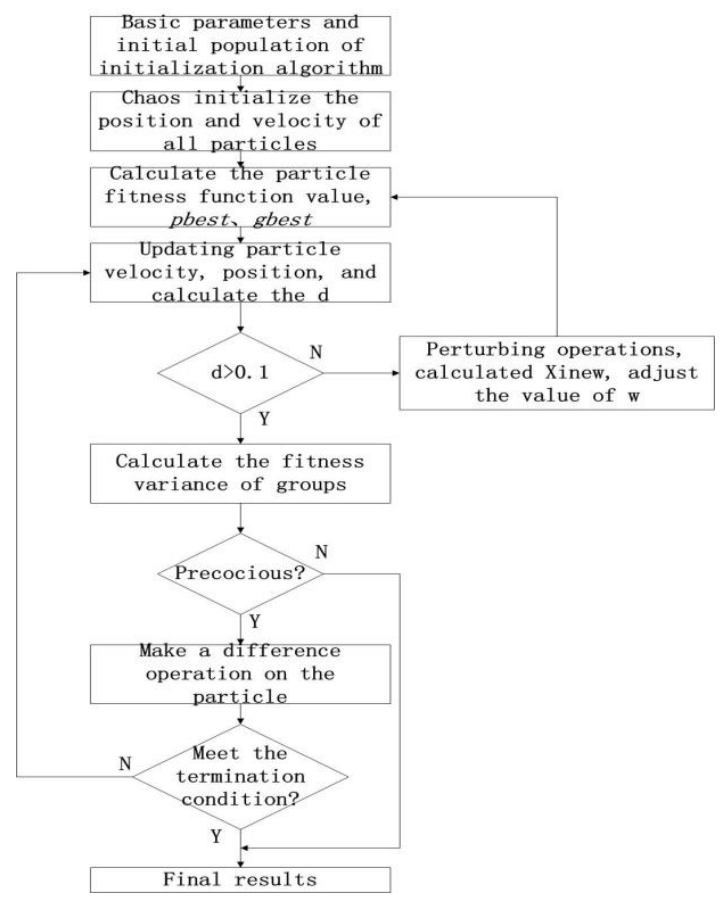

Figure 1: CDEDPSO algorithm flow chart

Test function and data analysis

In this paper,hybrid optimization algorithm is tested by four standard test function.They are Griewank function,Rosenbrock function,Schwefel function and Rastrigin function.Griewank function and Rastrigin function is a multimodal function. They can test the global search performance of the algorithm effectively.Rosenbrock function can detect the speed of convergence effectively.Its global optimal hidden in a narrow passage and is not easy to obtain.Schwefel function is a high-dimensional unimodal function and can examine the convergence speed and accuracy of the algorithm $^{[12]}$.

The range of the independent variable of Griewank function is [-600, 600], the dimension is 10 , the function theory optimal value is 0 .The range of the independent variable of Rosenbrock function is $[-30,30]$, the dimension is 10 , the function theory optimal value is 0 . The range of the independent variable of Schwefel function is $[-10,10]$, the dimension is 30 , the function theory optimal value is 0 .The range of the independent variable of Rastrigin function is [-5.12,5.12], the dimension is 10,the function theory optimal value is $0^{[13]}$.

parameter settings in hybrid optimization algorithm are as follows: the values of $v_{\max }$ and $v_{\min }$ are 0.1 times the upper limit and lower limit of position. $\omega_{\max }=0.9, \omega_{\min }=0.4, c_{1}=c_{2}=2.0$.All algorithms are all population size 30 .Each test is set to run 10 times and the average value is as the final result.Table 1 shows the test results of four test functions,including the average 'mean', standard deviation 'std', The average number of iterations ' $\mathrm{k}$ ', that is to run 10 times to get the high-quality solutions, the number of finding the quality solutions ' $n$ '. 
Table 1 Test results of three algorithms for the four standard test function

\begin{tabular}{|c|c|c|c|c|c|c|c|c|c|c|c|c|}
\hline \multirow{2}{*}{ Function } & \multicolumn{4}{|c|}{ PSO } & \multicolumn{4}{|c|}{ DEPSO } & \multicolumn{4}{|c|}{ CDEDPSO } \\
\hline & mean & std & $\mathrm{k}$ & $\mathrm{n}$ & mean & std & $\mathrm{k}$ & $\mathrm{n}$ & mean & std & $\mathrm{k}$ & $\mathrm{n}$ \\
\hline $\begin{array}{c}\text { Griewan } \\
\mathrm{k}\end{array}$ & $\begin{array}{c}3.7832 \mathrm{e}- \\
01\end{array}$ & $\begin{array}{c}1.1013 \mathrm{e}- \\
01\end{array}$ & $>400$ & 2 & $\begin{array}{c}1.2623 \mathrm{e}- \\
02\end{array}$ & $\begin{array}{c}1.8347 \mathrm{e}-0 \\
5\end{array}$ & $\begin{array}{c}>20 \\
0\end{array}$ & 3 & $\begin{array}{c}5.4157 \mathrm{e}- \\
10\end{array}$ & $\begin{array}{c}3.3473 \mathrm{e}- \\
10\end{array}$ & $\begin{array}{c}>10 \\
0\end{array}$ & 4 \\
\hline $\begin{array}{c}\text { Rosenbro } \\
\text { ck }\end{array}$ & 22.9198 & 19.5505 & $>2000$ & 1 & 3.74223 & $\begin{array}{c}8.6673 \mathrm{e}-0 \\
1\end{array}$ & $\begin{array}{c}>12 \\
00\end{array}$ & 3 & 0.1246 & $\begin{array}{c}2.0624 \mathrm{e}- \\
03\end{array}$ & $\begin{array}{c}>10 \\
00\end{array}$ & 5 \\
\hline Schwefel & 1.3247 & $\begin{array}{c}3.4875 \mathrm{e}- \\
02\end{array}$ & $>300$ & 2 & $\begin{array}{c}8.906 \mathrm{e}-0 \\
1\end{array}$ & $\begin{array}{c}1.15681 \mathrm{e}- \\
04\end{array}$ & $\begin{array}{c}>20 \\
0\end{array}$ & 4 & $\begin{array}{c}1.123 \mathrm{e}-0 \\
2\end{array}$ & $\begin{array}{c}2.7608 \mathrm{e}- \\
06\end{array}$ & $\begin{array}{c}>15 \\
0\end{array}$ & 6 \\
\hline Rastrigin & 1.1400 & 1.2337 & $>500$ & 3 & $\begin{array}{c}5.793 e-0 \\
2\end{array}$ & $\begin{array}{c}8.3142 \mathrm{e}-0 \\
3\end{array}$ & $\begin{array}{c}>45 \\
0\end{array}$ & 3 & 0 & $\begin{array}{c}4.9547 \mathrm{e}- \\
06\end{array}$ & $>80$ & 4 \\
\hline
\end{tabular}

Figure 2 shows the optimization graph of four test functions.The $\mathrm{Y}$-axis is fitness value.According to the test function optimal value,optimized fitness value tends to 0 . The $\mathrm{X}$-axis is the number of iterations to optimize. The evolution curve of four test functions are shown in Figure 2:

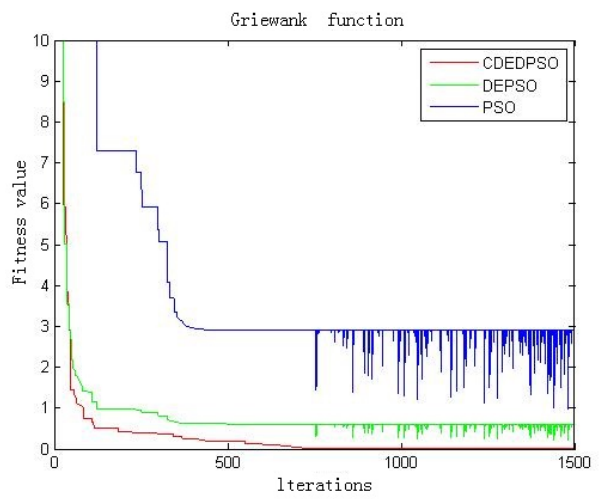

(a)

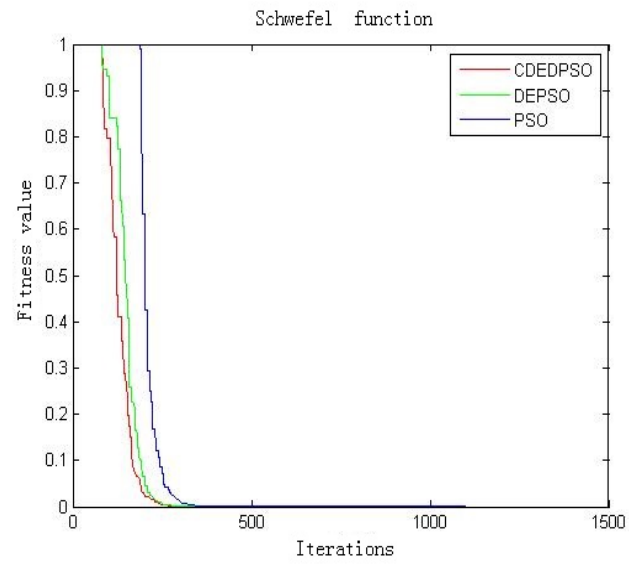

(c)

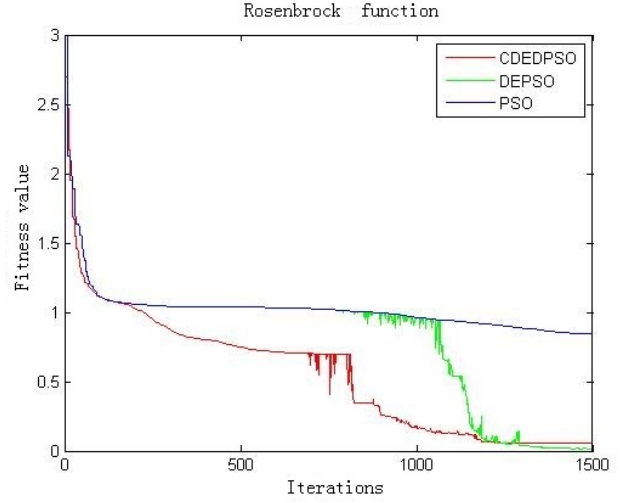

(b)

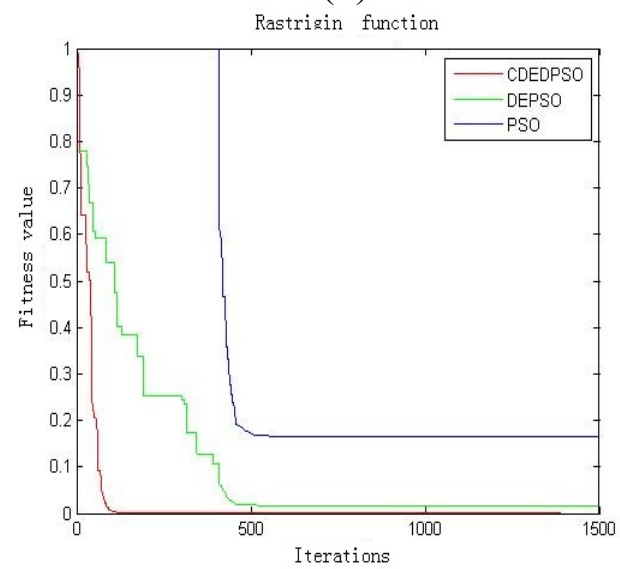

(d)

Figure 2: Evolution curve of four test functions

The average (mean) may reflect the convergence accuracy, the smaller the average value, the higher the convergence precision; The value of standard deviation (std) represents the strength of the robustness of the algorithm. The smaller the standard deviation, the stronger robustness. Convergence of the algorithm is reflected on the value of iterations $(\mathrm{k})$. The fewer the number of iterations, the better the convergence of the algorithm;Then $\mathrm{n}$ reflects the global search ability of the algorithm,the larger the value of $\mathrm{n}$, the stronger global search ability of the algorithm.For the four test functions, in the terms of mean,std,k and n,CDEDPSO algorithm is superior to PSO algorithm and DEPSO algorithm according to figure 2 and table 1.And it is reflected in the evolution curve visually.Therefore,the optimization algorithm proposed in this paper is better than the traditional 
DEPSO algorithm obviously in terms of accuracy, convergence speed,robustness, global search ability.

\section{CDEDPSO algorithm in FPN model}

FPN uncertain knowledge representation model

A FPN uncertain knowledge representation rules are described below:

Rule 1 if $p_{1}$ and $p_{2}$ and $p_{3}$ and $p_{4}$ then $p_{10}\left(\lambda_{1}, \mu_{1}, \omega_{1}, \omega_{2}, \omega_{3}, \omega_{4}\right)$;

Rule 2 if $p_{4}$ and $p_{5}$ and $p_{6}$ then $p_{11}\left(\lambda_{2}, \mu_{2}, \omega_{5}, \omega_{6}, \omega_{7}\right)$;

Rule 3 if $p_{6}$ and $p_{7}$ then $p_{12}\left(\lambda_{3}, \mu_{3}, \omega_{8}, \omega_{9}\right)$;

Rule 4 if $p_{8}$ then $p_{13}\left(\lambda_{4}, \mu_{4}\right)$;

Rule 5 if $p_{8}$ and $p_{9}$ then $p_{14}\left(\lambda_{5}, \mu_{5}, \omega_{10}, \omega_{11}\right)$;

Rule 6 if $p_{10}$ then $p_{15}\left(\lambda_{6}, \mu_{6}\right)$;

Rule 7 if $p_{11}$ then $p_{16}\left(\lambda_{7}, \mu_{7}\right)$;

Rule 8 if $p_{12}$ and $p_{13}$ then $p_{17}\left(\lambda_{8}, \mu_{8}, \omega_{12}, \omega_{13}\right)$;

Rule 9 if $p_{14}$ then $p_{18}\left(\lambda_{9}, \mu_{9}\right)$.

Among them, $\omega_{j}, \mu_{i}, \lambda_{k}(j, i, k \in N)$ represent the weights, certainty and threshold.Based on the rules above,establish the FPN model and is shown in Figure 3:

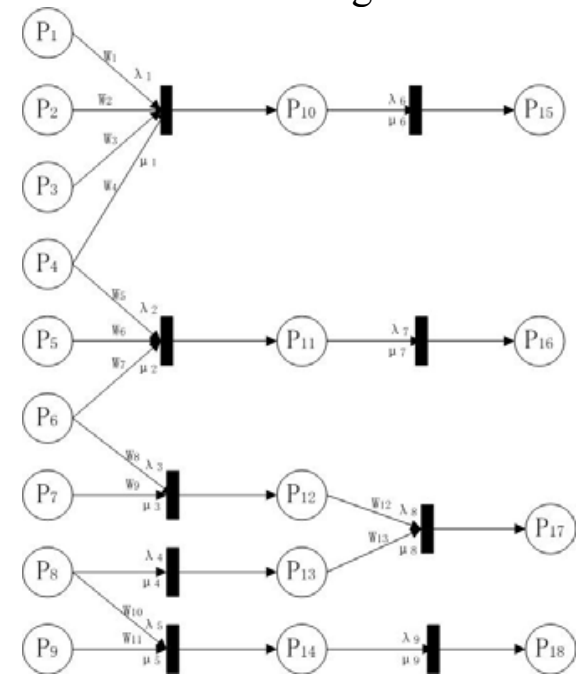

Figure 3: Uncertainty knowledge representation FPN model

Optimize FPN model parameters

Based on the optimization algorithm proposed by this paper,using the Griewank test function to do self-learning for the uncertain parameters in FPN uncertain knowledge representation model.And it can improve the value of uncertain parameters. The results obtained are shown in tables 4 to 6: 
Table 4 Comparison of weight $\omega$ error values (mean) that the output before and after optimization

\begin{tabular}{cccccccccccccc}
\hline Parameter & $\omega_{1}$ & $\omega_{2}$ & $\omega_{3}$ & $\omega_{4}$ & $\omega_{5}$ & $\omega_{6}$ & $\omega_{7}$ & $\omega_{8}$ & $\omega_{9}$ & $\omega_{10}$ & $\omega_{11}$ & $\omega_{12}$ & $\omega_{13}$ \\
\hline PSO & 2.0 & 4.2 & 4.5 & 2.1 & 3.2 & 4.8 & 5.1 & 6.6 & 3.6 & 7.1 & 4.8 & 5.5 & 7.4 \\
$(\mathrm{e}-04)$ & & & & & & & & & & & & & \\
CDEDPS & 1.2 & 0.9 & 2.9 & 1.3 & 0.3 & 1.9 & 0.6 & 1.2 & 0.8 & 1.3 & 0.9 & 1.6 & 1.5 \\
$0 \quad(\mathrm{e}-05)$ & & & & & & & & & & & & &
\end{tabular}

Table 5 Comparison of certainty $\mu$ error values (mean) that the output before and after optimization

\begin{tabular}{cccccccccc}
\hline $\begin{array}{c}\text { Paramet } \\
\text { er }\end{array}$ & $\mu_{1}$ & $\mu_{2}$ & $\mu_{3}$ & $\mu_{4}$ & $\mu_{5}$ & $\mu_{6}$ & $\mu_{7}$ & $\mu_{8}$ & $\mu_{9}$ \\
\hline $\begin{array}{c}\text { PSO } \\
\text { (e-04) }\end{array}$ & 9.1 & 7.4 & 8.5 & 8.7 & 7.6 & 8.6 & 9.9 & 8.3 & 9.8 \\
CDEDP & 0.8 & 3.1 & 1.6 & 2.7 & 0.5 & 3.4 & 1.7 & 1.2 & 2.9 \\
$\begin{array}{c}\text { SO } \\
(\mathrm{e}-05)\end{array}$ & & & & & & & & & \\
\hline
\end{tabular}

Table 6 Comparison of threshold $\lambda$ error values (mean) that the output before and after optimization

\begin{tabular}{cccccccccc}
\hline $\begin{array}{c}\text { Paramet } \\
\text { er }\end{array}$ & $\lambda_{1}$ & $\lambda_{2}$ & $\lambda_{3}$ & $\lambda_{4}$ & $\lambda_{5}$ & $\lambda_{6}$ & $\lambda_{7}$ & $\lambda_{8}$ & $\lambda_{9}$ \\
\hline PSO & 5.4 & 4.8 & 3.2 & 6.2 & 4.3 & 4.2 & 3.8 & 4.1 & 2.9 \\
$\left(\begin{array}{l}\text { e-04) } \\
\text { CDEDP }\end{array}\right.$ & 2.3 & 0.5 & 1.9 & 0.4 & 2.3 & 1.0 & 1.9 & 0.1 & 1.1 \\
SO & & & & & & & & & \\
$(\mathrm{e}-05)$ & & & & & & & & & \\
\hline
\end{tabular}

As can be seen from table 4-6,optimization algorithm proposed by this paper makes error value of each parameter in FPN model is smaller. Therefore, use the optimized FPN model to do reasoning, the result is more accurate.

\section{Conclusions}

For the change characteristics of uncertain parameters in some large complex systems, this paper presents a FPN model method based on CDEDPSO optimization algorithm. This method makes full use of the convergence of PSO algorithm and the global search ability of DE algorithm and to improve.It makes up the defect that the uncertainty of weights, thresholds, certainty and other parameters in traditional FPN model.Finally, apply the method to some FPN model to verify its accuracy and efficiency.

\section{References}

[1]Peng Xun, Wang Wei-Ming,Gu Chao-Chen,Hu Jie.Representation Method of Dynamic Uncertain Knowledge Based on Fuzzy-Petri Net and Genntic-PSO Algorithm[J].PR\&AI,Vol.27(2014),p123 128.

[2]Gong Mao-fa,Zhang Yan-pan,Liu Yan-ni.Fault Diagnosis of Power Transformers Based on Back Propagation Algorithm Evolving Fuzzy Petri Nets[J].Power System Protection and 
Control,Vol.43(2015),p22 26.

[3]Zhang Chao,Chao Peng-jun,Zhang jie-xin. Fault Diagnosis of Hydraulic System of Concrete Pump Based on Fuzzy Petri Network[J].Equipment Management and Maintenance,Vol.32(2015),p87-91.

[4]Sandre-Hernandez,O.,Morales-Caporal,R.,Rangel-Magdaleno,J.,Peregrina-Barreto,H.,Hernande z-Perez,J.N.Parameter Identification of PMSMs Using Experimental Measurements and a PSO Algorithm[J].Instrumentation and Measurement,IEEE Transactions on,Vol.64(2015),p56 65.

[5]Leitão,H.A.S.,Lopes,W.T.A.,Madeiro,F.,PSO Algorithm Applied to Codebook Design for Channel-Optimized Vector Quantization[J],Latin America Transactions, IEEE (Revista IEEE America Latina),Vol.13(2015),p89 93.

[6]Silva,E.H.M.,Bastos Filho,C.J.A.PSO Efficient Implementation on GPUs Using Low Latency Memory[J].Latin America Transactions,IEEE (Revista IEEE America Latina),Vol.13(2015),p167 175.

[7]Samani,M.,Tafreshi,M.,Shafieenejad,I.,Nikkhah,A.A..Minimum-time open-loop and closed-loop optimal guidance with GA-PSO and neural fuzzy for Samarai MAV flight[J].Aerospace and Electronic Systems Magazine,IEEE,Vol.30(2015),p189 194.

[8]Fei,R.,Moreau,J.,Mir,S.,Marcellin,A.,Mandier,C.;Huss,E.,Palmigiani,G.,Vitrou,P.,Droniou,T..H orizontal-FPN fault coverage improvement in production test of CMOS imagers[J].VLSI Test Symposium (VTS),IEEE Vol.33(2015),p167 173.

[9]Chen,S.-J.,Zhan,T.-S.,Huang,C.-H.,Chen,J.-L.,Lin,C.-H..Nontechnical Loss and Outage Detection Using Fractional-Order Self-Synchronization Error-Based Fuzzy Petri Nets in Micro-Distribution Systems[J].Smart Grid,IEEE Transactions on, Vol.6(2015),p138 143.

[10]Wei-Ling Chen,Chung-Dann Kan,Chia-Hung Lin,Tainsong Chen,A Rule-Based Decision-Making Diagnosis System to Evaluate Arteriovenous Shunt Stenosis for Hemodialysis Treatment of Patients Using Fuzzy Petri Nets[J].Biomedical and Health Informatics,IEEE Journal of,Vol.18(2014),p82 89.

[11]Gniewek,L.,Coverability Graph of Fuzzy Interpreted Petri Net[J].Systems, Man, and Cybernetics:Systems, IEEE Transactions on,Vol.44(2014),p34 37.

[12]Kouquan Zheng, Yingjie Lei,Rui Wang, Yi Wang, Xiaoyong Shen.Method for parameters optimization of IFPN based on BP algorithm[J]. Control and Decision,Vol.28(2013),p45.(In Chinese).

[13]Tao Wu, Yu-song Yan, Xi Chen.Improved dual-group interaction QPSO algorithm based on random evaluation[J]. Control and Decision,Vol.30(2015),p.63.(In Chinese) 